\title{
Characterization of platelet functionality in pediatric patients with kaposiform hemangioendothelioma / Kasabach-Merritt phenomenon
}

\author{
Alexey Martyanov ${ }^{1}$, Ivan Tesakov ${ }^{1}$, Olga An², Julia-Jessica Korobkin ${ }^{3}$, Anastasia \\ Ignatova $^{1}$, Galina Svidelskaya ${ }^{3}$, Eugenia Yushkova ${ }^{4}$, Ekaterina Koltsova ${ }^{3}$, Nadezhda \\ Podoplelova $^{3}$, Galina Novichkova ${ }^{1}$, Lili Khachatryan ${ }^{1}$, Johannes Eble ${ }^{5}$, Dmitrii Kalinin ${ }^{6}$, \\ and Anastasia Sveshnikova ${ }^{3}$ \\ ${ }^{1}$ Dmitry Rogachev National Medical Research Center of Pediatric Hematology Oncology \\ and Immunology \\ ${ }^{2}$ Sechenov University \\ ${ }^{3}$ Center for Theoretical Problems of Physico-Chemical Pharmacology RAS \\ ${ }^{4}$ Pirogov Russian National Research Medical University \\ ${ }^{5}$ University of Münster Institute of Physiological Chemistry and Pathobiochemistry \\ ${ }^{6}$ University of Münster Department of Chemistry and Pharmacy
}

December 14, 2021

\begin{abstract}
Background. Kaposiform hemangioendothelioma (KHE) is a rare vascular tumor of infancy commonly associated with Kasabach-Merritt phenomenon (KMP) that includes thrombocytopenia and coagulation dysfunction. Platelet receptor CLEC2 -tumor cell podoplanin interaction is considered the key mechanism of thrombocytopenia in KMP, however, the effect of long-term exposure to podoplanin on platelet function is unknown.

Procedure. Here we examined blood samples from 7 patients with KHE/KMP. Platelet calcium signaling and functional responses to conventional activation and CLEC-2 stimulation were analyzed by continuous and endpoint live cell flow cytometry. Platelet aggregation in response to ADP or rhodocytin was analyzed by low-angle light scattering approach (LaSca). Additionally, ex vivo thrombus formation on collagen was observed in parallel-plate flow chambers.

Results. We demonstrate that in KHE/KMP platelet functional responses to strong stimulation were on the lower boundary of age-matched normal ranges, while calcium mobilization and fibrinogen binding upon stimulation with ADP alone were significantly lower than control values. Platelet di-aggregate formation in response to ADP was also diminished in most of the patients. Formation of platelet aggregates in collagen-coated parallel plate flow chambers was also noticeably lower than in the age-matched control group. Calcium mobilization in response to CLEC-2 stimulation was unaltered in the patients and could be blocked by low-molecular-weight inhibitors, 2CP and HB125.

Conclusions. While platelet responsiveness in KHE/KMP is moderately altered, platelet CLEC-2 receptors remain functional and respond to inhibition. Therefore, our findings suggest that CLEC-2-targeting molecules are new potential agents in therapeutic management of this life-threatening condition.
\end{abstract}

\section{Introduction}

Vascular neoplasms are tumors arising from blood vessel endothelial cells. Kaposiform hemangioendothelioma (KHE) is a rare (incidence $0.07: 100000$ per year $^{1}$ ) vascular tumor of infancy with locally aggressive 
behavior ${ }^{2}$. The most common KHE tumor sites are limbs and the trunk, followed by the head and neck ${ }^{3}$. KHE is commonly $\left(71 \%\right.$ cases $^{1}$ ) associated with the Kasabach-Merritt phenomenon (KMP), a potentially life-threatening hematologic complication ${ }^{4}$ with reported mortality of up to $30 \%^{1}$. KMP refers to severe thrombocytopenia, consumptive coagulation dysfunction, secondary fibrinogen reduction, and microangiopathic hemolytic anemia ${ }^{5}$. Histologically, KHE is composed of infiltrating nodules with slit-like or crescentic vessels lined by spindled endothelial cells ${ }^{6}$. When they are dilated, also lymphatic channels are observed, this is called «kaposiform lymphangiomatosis» ${ }^{7}$. KHE is primarily positive for endothelial markers CD31 and CD34 and lymphatic markers LYVE1, PROX1, and podoplanin ${ }^{8}$. KMP causes platelet «trapping» and activation within abnormal tumor vessels, which results in formation of platelet thrombi and initiaton of plasma coagulation ${ }^{9}$. The subsequent hyper-activation of the fibrinolytic system may cause intratumoral hemorrhage ${ }^{6,10}$. Unlike DIC (disseminated intravascular coagulation), KMP causes localized coagulation disorder. However, DIC may be eventually induced in severe cases of KMP ${ }^{10}$.

Despite these findings, the etiology of KMP and the initial molecular mechanisms of platelet activation in KHE are not fully characterized. KHE cells are known to express podoplanin, the only known endogenous ligand for the platelet receptor CLEC-2 ${ }^{11}$. The critical physiological function of podoplanin-induced platelet aggregation is the embryological separation of blood and lymphatic circulations ${ }^{11,12}$. CLEC-2 also contributes to vascular integrity during inflammation ${ }^{13,14}$ and plays a role in thrombosis and wound healing 15,16. Thus, in patients with podoplanin-expressing KHE, the long-term platelet exposure to podoplanin might potentially affect platelet functionality.

Upon activation in KHE, platelets secrete their granule content, which can cause tumor growth and progression ${ }^{17}$. Clinical findings support the critical role of platelets in KHE progression and development, namely, platelet transfusions in patients with KMP can lead not only to rapid tumor growth but also to severe deterioration of the coagulopathy ${ }^{18,19}$. While platelet activation is considered to be one of the critical pathogenic mechanisms in KMP, antiplatelet therapy for patients with KMP did not give positive results ${ }^{20}$. Moreover, usage of antiplatelet agents for patients with severe thrombocytopenia is controversial ${ }^{21,22}$. Therefore, understanding platelet functioning in KHE/KMP is critically important in therapeutic management of these conditions.

In this study, we examined platelets from 7 patients with KHE/KMP. We found that in KHE, platelets are less responsive to weak stimulation, while platelet responsiveness to strong stimulation is mostly unimpaired.

\section{Methods}

\section{Patients and blood collection}

We enrolled patients who received treatment in Dmitry Rogachev National Medical Research Center to this study. The key inclusion criteria were: the presence of a vascular tumor (kaposiform hemangioendothelioma or bundle angioma) and associated coagulopathy and thrombocytopenia at diagnosis. Patients, who received platelet transfusions within 3 weeks prior to enrolment, were excluded from the study. Age and treatment history were not exclusion criteria. Age-matched healthy volunteers were recruited as controls to this study.

At the enrollment, $1.6 \mathrm{~mL}$ of blood were collected into hirudin containing S-Monovette(r) tubes, and $4.3 \mathrm{~mL}$ of blood were collected into sodium citrate containing S-Monovette(r) tubes (Monovette, Sarstedt, Newton, NC). Blood samples were processed within 1.5 hours after collection.

The study protocol was approved by the independent ethics committee of the CTP PCP RAS (approval number 3/1-21, 05.10.2021). All participants provided written informed consent before enrolment. The study was conducted in accordance with the principles of the Declaration of Helsinki and the International Conference on Harmonization Good Clinical Practice Guideline. Data available on request from the authors. 


\section{Materials}

The materials were as follows: calcium-sensitive cell-permeable fluorescent dye Fura-Red-AM, (Molecular Probes, Eugene, USA); ADP, EGTA, HEPES, bovine serum albumin, apyrase grade VII, TRAP (SFLLRN), AYGPKF, mepacrine (Sigma-Aldrich, St Louis, USA); CD62p-Alexa647, CD42b-PE, CD61-Alexa647, PAC1FITC (Sony Biotechnology, San Jose, USA), collagen (NPO Renam, Moscow, Russia). Cysteine-containing version of cross-linked collagen-related peptide (CRP) was kindly provided by Prof. R.W. Farndale (the University of Cambridge, Cambridge, UK). Rhodocytin was isolated as previously described ${ }^{23,24(\mathrm{p})}$. CLEC2 inhibitors $2 \mathrm{CP}^{25}$ and HB125 were synthesized as described in Supporting information.

Tyrode's buffer $\left(150 \mathrm{mM} \mathrm{NaCl}, 2.7 \mathrm{mM} \mathrm{KCl}, 1 \mathrm{mM} \mathrm{MgCl}_{2}, 2 \mathrm{mM} \mathrm{CaCl}_{2}, 0.4 \mathrm{mM} \mathrm{NaH} \mathrm{PO}_{4}, 0.4 \mathrm{mM}\right.$ $\mathrm{Na}_{2} \mathrm{CO}_{3}, 5 \mathrm{mM}$ HEPES, $5 \mathrm{mM}$ glucose, $0.5 \%$ BSA, $\mathrm{pH}$ 7.35) was freshly prepared from reagents (SigmaAldrich, St Louis, MO).

\section{Endpoint flow cytometry}

Functional testing of the platelets was performed as described ${ }^{26}$. Briefly, blood was collected into sodium citrate $(3.8 \% \mathrm{v} / \mathrm{v})$ vacuum tubes and then diluted 20 times by Tyrode's buffer without $2.5 \mathrm{mM}$ calcium. Samples were then incubated 1:1 with activators $(12.5 \mu \mathrm{M}$ SFLLRN and $10 \mu \mathrm{g} / \mathrm{mL}$ CRP) or $(12.5 \mu \mathrm{M}$ SFLLRN, $200 \mu \mathrm{M}$ AYGPKF and $2 \mu \mathrm{M}$ ADP) or vehicle in Tyrode's-Calcium (5 mM) buffer. After 10 min incubation, samples were mixed 1:1 with anti-CD42b, anti-CD61, PAC1, anti-CD62p, and Annexin-V in Tyrode's-Calcium $(2.5 \mathrm{mM})$ buffer and left for 10 minutes. Alternatively, platelets were incubated with 10 $\mu \mathrm{M}$ mepacrine for 30 minutes. After this, samples were diluted to the platelet concentration of $5000 \mathrm{plt} / \mu \mathrm{L}$ by Tyrode's Calcium $(2.5 \mathrm{mM})$ buffer and studied using NovoCyte Acea Flow cytometer.

\section{Continuous flow cytometry}

Assays of platelet signaling were performed as described ${ }^{27}$. Hirudinated whole blood was incubated with $2 \mu \mathrm{M}$ of Fura-Red in the presence of $1 \mathrm{U} / \mathrm{mL}$ of apyrase for 35 minutes at $37^{\circ} \mathrm{C}$. Leukocyte rich plasma (LRP) was collected, diluted by the Tyrode's-Calcium buffer to the final concentration of $1000 \mathrm{plt} / \mu \mathrm{L}$, and left resting for 30 minutes. $100 \mu \mathrm{L}$ was the lowest LRP volume used in this study. $100 \mu \mathrm{g} / \mathrm{mL}$ of Alexa488 labeled human fibrinogen was added 2 minutes before sample loading to the BD FACS Canto II Flow Cytometer (BD Biosciences, San Jose, US). Samples were analyzed in a continuous mode. The primary fluorescence signal was averaged over equal periods. The ratio of calcium bound Fura-Red (excited by 405 $\mathrm{nm}$ laser) to calcium-free Fura-Red (excited by $488 \mathrm{~nm}$ laser) was recalculated into platelet cytosolic calcium concentration using Grynkiewicz formula ${ }^{28}$.

\section{Laser-scanning aggregometry}

Low angle scattering analysis of platelet activation was performed as described elsewhere ${ }^{29}$. Briefly, LaSca method is based on the measurement of low angle light scattering in a diluted platelet suspension. This method is used to characterize the platelets function, allowing simultaneous recording of platelet di-aggregate formation (light scattering at $1.5^{\circ}$ ) and shape changing (light scattering at $12^{\circ}$ ). Platelet-rich plasma was diluted by Tyrode's buffer to platelet concentration $10000 \mathrm{plt} / \mu \mathrm{L}$, and the initial velocity and aggregation amplitude upon platelet stimulation were evaluated.

Immunofluorescence microscopy of blood smears

Immunofluorescence labelling of standard air-dried peripheral blood smears with antibodies against glycoprotein (GP)Ib, GPIIb, von Willebrand factor (VWF), P-selectin, lysosomal-associated membrane protein (LAMP)-1, LAMP-2, LAMP-3, non-muscle myosin IIA (NMMIIa), $\beta 1$-tubulin, was performed as described by A. Greinacher and others ${ }^{30}$. 
Ex vivo thrombus growth analysis

Ex vivo thrombus growth analysis was conducted as described previously ${ }^{27,31}$. Briefly, hirudinated whole blood was preincubated with DiOC-6 (500 nM) and perfused through the parallel plate flow chambers with fibrillary collagen $(100 \mu \mathrm{g} / \mathrm{mL})$ covered glass at $100 \mathrm{~s}^{-1}$ shear rate. Thrombus growth was observed using a Nikon Eclipse Ti-E inverted microscope (Tokyo, Japan).

Thrombodynamics assay

Thrombodynamics (TD) was performed using a Thrombodynamics Analyzer and Thrombodynamics kit (LLC HemaCore, Moscow, Russia) as previously described ${ }^{32}$. This method is based on registering spatial fibrin clot growth after activation of clotting in a thin layer of plasma after contact with an immobilized tissue factor bearing surface.

Statistical analysis

Flow cytometry data were analyzed by means of FlowJo (https://www.flowjo.com/) and Python 3.8. Aggregation data were analyzed using Python 3.8. Statistical analysis of the data was performed by means of Python 3.8. The data were analyzed using nonparametric Mann-Whitney U test and Wilcoxon signed-rank test. The significance level was set as $95 \%$.

\section{Results}

\section{Patient characteristics}

7 patients were enrolled to the study. Most of them (71\%) were male, and the median age at enrollment was 11 months. The most common tumor site was soft tissues of the upper body, and the disease predominantly manifested since newborn with a deep thrombocytopenia and an associated coagulopathy (KMP). Biopsy demonstrated that $6 / 7$ patients had KHE, and one patient (Patient 4) had kaposiform lymphangiomatosis according to histological examination. In $5 / 7$ cases, immunohistochemistry was performed and revealed positive staining for podoplanin, CD31, and CD34.

None of the subjects was treatment-naïve. Six patients had a history of prior KMP at disease manifestation, but had adequate blood cell counts at enrollment. One patient (Patient 1) presented with KMP (thrombocytopenia, severe coagulopathy) at the time of enrollment. Patients 1, 2, 4, and 5 received cyclophosphamide and vinblastine with/without liposomal doxorubicin (LD), Patient 7 received LD and vincristine, Patient 6 received LD and sirolimus, Patient 3 received sirolimus and hydrocortisone. Two patients also received $\beta$-blockers: propranolol (Patient 2), or atenolol (Patient 7). All clinical, laboratory, and treatment data are summarized in Table S1.

\section{Platelet activation in response to strong stimulation}

Based on the fact that KHE/KMP pathogenesis is partially based on platelet-tumor interactions, we expected altered platelet functionality in KHE. Therefore, we studied platelet functional responses (shape change, GPIb shedding, granule release, integrin activation and phosphatidylserine exposure) in response to a 10 minstimulation with either combination of CRP (GPVI agonist) and SFLLRN (PAR1 agonist), or combination of AYGPKF (PAR4 agonist), SFLLRN and ADP, by endpoint flow cytometry (Fig. 1).

Altogether, platelet responses to strong activation were within normal ranges (Fig. 1). For P1 and P4 an increased FSC MFI was observed (Fig. 1A). For P1, this could be explained by inflammation related increased platelet consumption, while for $\mathrm{P} 4$ this could be an effect of tubulin ring disruption by vinblastine, also observed in microscopy (Fig. S2). Platelet integrin activation in response to thrombin and purinergic receptors stimulation ("2TR+ADP" in Figure 1) was significantly diminished in KHE patients (Fig. 1B), while other platelet responses to activation were on the lower boundary of those of healthy donors. P1 alone 
demonstrated significantly altered platelet responses, which is consistent with DIC-like phenotype of this patient.

Immunofluorescence microscopy of peripheral blood smears was performed in 5/7 patients (Patients 3-7) and revealed normal platelet staining for GPIb, GPIIb, VWF, P-selectin, LAMP-1, -2, -3, and NMMIIa in all the patients (data not shown). In patients 4,5 , and 7 abnormal diffuse distribution of platelet $\beta 1$-tubulin was observed (Fig. S2). This phenomenon could be explained by treatment with tubulin-targeted agents, vincristine or vinblastine.

Ex vivo platelet aggregation and blood plasma coagulation

4/7 patients (Patients 2, 3,6 and 7) demonstrated almost normal blood plasma coagulation. P1 demonstrated significant hypercoagulation, and Patients 4,5 and 6 demonstrated mild hypocoagulation (Fig. S1). These data are consistent with the presence of KMP and DIC-like phenotype in P1 and the previously reported reduction of D-dimer levels upon sirolimus administration ${ }^{33}$. Altogether we conclude that KHE does not significantly affect blood coagulation.

Ex vivo platelet aggregation in collagen covered parallel-plate flow chambers was performed as described previously ${ }^{27}$. In accordance with previously published results, thrombi in the samples from the thrombocytopenic patient (P1) were significantly smaller than those of healthy donors (Fig. 2A). However, thrombi formed in the samples of patients with KHE (non-thrombocytopenic) were also noticeably smaller than those from healthy donors' samples (Fig. 2B-D).

Platelet calcium signaling responses

While strong platelet activation in patients with KHE was mostly unimpaired, we decided to analyze the responses to rhodocytin ${ }^{23}$ and fucoidan ${ }^{34}$ in comparison with the conventional platelet activation (Fig. 3). The key intracellular signal that triggers platelet functional responses is cytosolic calcium concentration ${ }^{35}$, while platelet shape change and reversible fibrinogen binding are the most sensitive platelet responses ${ }^{27,36}$.

Cytosolic calcium concentration in quiescent state was significantly lower in KHE samples (Fig. 3A). Calcium mobilization in response to $2 \mu \mathrm{M}$ ADP was also decreased in comparison to healthy controls (Fig. 3B). Fibrinogen-binding responses (Fig. 3C) were diminished in some of the patients. The most severe platelet dysfunction was observed again in P1. Impaired platelet responses to stimulation in KHE could possibly be explained by general dysfunction of platelet signaling associated with CLEC-2 malfunctioning. For patients with KHE we observed unaltered calcium mobilization upon stimulation with rhodocytin or fucoidan (Fig. $3 \mathrm{~A})$. However, in the presence of CLEC-2 inhibitors 2CP $(2 \mu \mathrm{M})$ and HB125 $(2 \mu \mathrm{M})$, calcium responses to rhodocytin (Fig. 3A) were significantly reduced in KHE samples; while the impact of these inhibitors was less pronounced for samples from healthy donors. Interestingly, being administered at $2 \mu \mathrm{M}, 2 \mathrm{CP}$ and HB125 themselves induced calcium mobilization in platelets comparably to rhodocytin $(200 \mathrm{nM})$ in KHE samples and higher than rhodocytin $(200 \mathrm{nM})$ in healthy controls (Fig. 3A). This might be related to partial agonistic properties of these CLEC-2 inhibitors which upon binding to the podoplanin/rhodocytin binding site on CLEC-2 could trigger its activation to some extent, especially, when administered at a high dose.

\section{Platelet di-aggregation}

In order to understand the functional significance of the observed decrease in platelet signaling responses in KHE (Fig. 3), aggregometry studies with weak platelet stimulation were performed. While thrombocytopenia is commonly observed in KHE/KMP, LaSca aggregometry was performed, as this method requires low platelet concentrations in probe (Fig. 4).

Typical plots of overall aggregation of platelets in KHE upon stimulation with $800 \mathrm{nM}$ ADP (black curves) and $20 \mu \mathrm{g} / \mathrm{mL}$ CRP (red curves) are shown in Figure 4A. In most of the patients, diminished initial velocity of aggregation upon stimulation with ADP (Fig. 4B) was observed, while maximal aggregation was mostly unimpaired (Fig. 4C). On the other hand, all the parameters of collagen-induced platelet aggregation were 
decreased in patients with KHE (Fig. 4D,E). These data are in line with previous findings on the reduced platelet responsiveness to stimulation with collagen (Fig. 1) as well as the impaired weak activation in KHE/KMP (Fig. 3). LaSca assays demonstrated that both 2CP and HB125 could cause decrease in initial velocity of aggregation (Fig. $4 \mathrm{~F}$ ) upon weak stimulation with $10 \mathrm{nM}$ rhodocytin. However, the inhibitors had no effect on maximum aggregation in patients with KHE (Fig. 4G).

\section{Discussion}

In this study, platelet responsiveness to conventional and CLEC-2-induced activation for patients with a podoplanin-expressing vascular tumor (KHE/KMP) was characterized. We found that platelet functionality and platelet aggregation tends to be impaired in patients with KHE and is significantly impaired in the patient with KMP (Fig. 1). Platelet integrin activation measured both by PAC-1 binding, fibrinogen binding and aggregation appears to be the most affected parameter (Fig. 1B, Fig. 2, Fig. 3B, Fig. 4B,D). Surprisingly, platelet calcium mobilization in response to CLEC-2 stimulation was unaltered in the patients' samples and we found that two novel low molecular weight CLEC-2 inhibitors successfully block platelet activation by rhodocytin (Fig. 3A, Fig. 4).

While podoplanin is considered the key platelet activator in KHE/KMP, we analyzed platelet responses to CLEC-2 agonists rhodocytin and fucoidan. Platelet cytosolic calcium increased normally upon stimulation with rhodocytin or fucoidan in KHE/KMP (Fig. 3A). Interestingly, either of the inhibitors of CLEC-2podoplanin interaction, $2 \mathrm{CP}$ or HB125, reduced noticeably calcium mobilization in response to rhodocytin, with more pronounced effect for KHE/KMP platelets. Moreover, aggregometry assay demonstrated that both 2CP and HB125 decrease initial velocity of aggregation in patients with KHE upon stimulation with rhodocytin. These findings suggest that CLEC-2 receptor and the components of CLEC-2:podoplanin downstream signaling pathway could be a promising target for pathogenetic therapy of KMP and related potentially life-threatening hematologic complications. To confirm this, furtherin vitro and in vivo studies are required.

Platelet CLEC-2 interaction with podoplanin is considered the key mechanism of platelet activation in KHE, but it may not be the only one involved in pathogenesis of KMP. Undoubtedly, KHE are tumors with extremely rapid growth and progression, and contain regions with impaired endothelial integrity, with exposure to tissue factor, VWF and collagen ${ }^{6}$, that can also lead to platelet activation, coagulation and thrombi formation. These additional mechanisms have been proven by positive tumor staining for VWF in $80 \% \operatorname{cases}^{37}$. Thus, further investigations are required to establish the biochemical basis of this phenomenon.

This study has several limitations. First, the study was conducted in a small cohort of patients. Despite the small sample size due to extreme rarity of KHE/KMP, the results obtained are still very valuable. The second limitation of this study is that the included patients are not treatment-naïve and most of them obtained hematologic response prior to enrollment. Thus, proposed hypotheses of this study require confirmation in a cohort of patients with newly diagnosed KHE/KMP.

\section{Conflict of Interest statement}

The authors declare no conflict of interest

\section{Acknowledgements}

The authors are grateful to Prof. Mikhail Panteleev (CTP PCP RAS) and Ms. Daria Morozova (Pirogov RNRMU) for valuable discussions on the subject. The authors want to thank Ms. Antonina Petlenko (CTP PCP RAS) and Ms. Evgenia Ponomarenko (NMRC PHOI) for the technical assistance during experiments. The study was supported by Russian Presidential Scholarship SP-2675.2019.4 for AAM and Russian Presidential Grants MK-432.2020.7 (for EMK) and MK-6271.2021.1.4 (for NAP); a grant from the endowment foundation "Science for Children" (for AAM and ANS) and Lomonosov Moscow State University Digital 
Medicine School. JAE is financially supported by the Interdisciplinary Center for Clinical Research (IZKF) Münster (grant: IZKF: Ebl-A/009/21). .

\section{References}

1. Croteau SE, Liang MG, Kozakewich HP, et al. Kaposiform hemangioendothelioma: Atypical features and risks of kasabach-merritt phenomenon in 107 referrals. Journal of Pediatrics . 2013;162(1):142-147. doi:10.1016/j.jpeds.2012.06.044

2. Mahajan P, Margolin J, Iacobas I. Kasabach-Merritt Phenomenon: Classic Presentation and Management Options. Clinical Medicine Insights: Blood Disorders . 2017;10:0-4. doi:10.1177/1179545X17699849

3. Chinello M, Di Carlo D, Olivieri F, et al. Successful management of kaposiform hemangioendothelioma with long-term sirolimus treatment: A case report and review of the literature. Mediterranean Journal of Hematology and Infectious Diseases . 2018;10(1):1-6. doi:10.4084/MJHID.2018.043

4. Enjolras O, Wassef M, Mazoyer E, et al. Infants with Kasabach-Merritt syndrome do not have "true" hemangiomas. Journal of Pediatrics . 1997;130(4):631-640. doi:10.1016/S0022-3476(97)70249-X

5. Kasabach H, Merritt K. Capillary Hemangioma With Extensive Purpura.American Journal of Diseases of Children . 1940;59(5):1063-1070. doi:10.1001/archpedi.1940.01990160135009

6. O'Rafferty C, O'Regan GM, Irvine AD, Smith OP. Recent advances in the pathobiology and management of Kasabach-Merritt phenomenon. British Journal of Haematology . 2015;171(1):38-51. doi:10.1111/bjh.13557

7. Zukerberg LR, Nickoloff BJ, Weiss SW. Kaposiform Hemangioendothelioma of Infancy and Childhood. The American Journal of Surgical Pathology . 1993;17(4):321-328. doi:10.1097/00000478-199304000-00001

8. Debelenko L V., Perez-Atayde AR, Mulliken JB, Liang MG, Archibald TH, Kozakewich HPW. D2-40 immunohistochemical analysis of pediatric vascular tumors reveals positivity in kaposiform hemangioendothelioma.Modern Pathology . 2005;18(11):1454-1460. doi:10.1038/modpathol.3800444

9. Seo SK, Suh JC, Na GY, Kim IS, Sohn KR. Kasabach-Merritt syndrome: Identification of platelet trapping in a tufted angioma by immunohistochemistry technique using monoclonal antibody to CD61.Pediatric Dermatology . 1999;16(5):392-394. doi:10.1046/j.1525-1470.1999.00103.x

10. Yao W, Li KL, Qin ZP, et al. Standards of care for Kasabach-Merritt phenomenon in China. World Journal of Pediatrics . 2021;17(2):123-130. doi:10.1007/s12519-020-00379-9

11. Suzuki-Inoue K, Inoue O, Ozaki Y. Novel platelet activation receptor CLEC-2: From discovery to prospects. Journal of Thrombosis and Haemostasis . 2011;9(1):44-55. doi:10.1111/j.1538-7836.2011.04335.x

12. Bertozzi CC, Schmaier AA, Mericko P, et al. Platelets regulate lymphatic vascular development through CLEC-2-SLP-76 signaling. Blood . 2010;116(4):661-670. doi:10.1182/blood-2010-02-270876

13. Gros A, Syvannarath V, Lamrani L, et al. Single platelets seal neutrophil-induced vascular breaches via GPVI during immune-complex-mediated inflammation in mice. Blood . 2015;126(8):1017-1026. doi:10.1182/blood-2014-12-617159

14. Rayes J, Lax S, Wichaiyo S, et al. The podoplanin-CLEC-2 axis inhibits inflammation in sepsis. Nature Communications . 2017;8(1). doi:10.1038/s41467-017-02402-6

15. Payne H, Ponomaryov T, Watson SP, Brill A. Mice with a deficiency in CLEC-2 are protected against deep vein thrombosis. Blood . 2017;129(14):2013-2020. doi:10.1182/blood-2016-09-742999

16. Inoue O, Hokamura K, Shirai T, et al. Vascular smooth muscle cells stimulate platelets and facilitate thrombus formation through platelet CLEC-2: Implications in atherothrombosis. PLoS ONE . 2015;10(9):128. doi:10.1371/journal.pone.0139357 
17. Klement GL, Yip TT, Cassiola F, et al. Platelets actively sequester angiogenesis regulators. Blood . 2009;113(12):2835-2842. doi:10.1182/blood-2008-06-159541

18. Phillips WG, Marsden JR. Kasabach-Merritt syndrome exacerbated by platelet transfusion. Journal of the Royal Society of Medicine . 1993;86(4):231-232. doi:10.1177/014107689308600418

19. Drolet BA, Trenor CC, Brandao LR, et al. Consensus-derived practice standards plan for complicated kaposiform hemangioendothelioma.Journal of Pediatrics . 2013;163(1):285-291. doi:10.1016/j.jpeds.2013.03.080

20. Koerper MA, Addiego JE, de Lorimier AA, Lipow H, Price D, Lubin BH. Use of aspirin and dipyridamole in children with platelet trapping syndromes. Journal of Pediatrics . 1983;102:311-314.

21. McCarthy CP, Steg G, Bhatt DL. The management of antiplatelet therapy in acute coronary syndrome patients with thrombocytopenia: a clinical conundrum. European Heart Journal . 2017;38(47):3488-3492. doi:10.1093/eurheartj/ehx531

22. Thachil J. Antiplatelet therapy - a summary for the general physicians. Clin Med . 2016;16(2):152-160. doi:10.7861/clinmedicine.16-2-152

23. Eble JA, Beermann B, Hinz HJ, Schmidt-Hederich A. $\alpha 2 \beta 1$ Integrin Is Not Recognized by Rhodocytin but Is the Specific, High Affinity Target of Rhodocetin, an RGD-independent Disintegrin and Potent Inhibitor of Cell Adhesion to Collagen. Journal of Biological Chemistry . 2001;276(15):12274-12284. doi:10.1074/jbc.M009338200

24. Suzuki-Inoue K. A novel Syk-dependent mechanism of platelet activation by the C-type lectin receptor CLEC-2. Blood . 2006;107(2):542-549. doi:10.1182/blood-2005-05-1994

25. Chang YW, Hsieh PW, Chang YT, et al. Identification of a novel platelet antagonist that binds to CLEC-2 and suppresses podoplanin-induced platelet aggregation and cancer metastasis. Oncotarget . 2015;6(40):42733-42748. doi:10.18632/oncotarget.5811

26. Ignatova AA, Ponomarenko EA, Polokhov DM, et al. Flow cytometry for pediatric platelets. Platelets . 2019;30(4):428-437. doi:10.1080/09537104.2018.1513473

27. Martyanov AA, Morozova DS, Sorokina MA, et al. Heterogeneity of integrin $\alpha \operatorname{Ib} \beta 3$ function in pediatric immune thrombocytopenia revealed by continuous flow cytometry analysis. International Journal of Molecular Sciences . 2020;21(9). doi:10.3390/ijms21093035

28. Grynkiewicz G, Poenie M, Tsien RY. A new generation of Ca2+ indicators with greatly improved fluorescence properties. Journal of Biological Chemistry . 1985;260(6):3440-3450. doi:10.1016/s00219258(19)83641-4

29. Mindukshev I, Gambaryan S, Kehrer L, et al. Low angle light scattering analysis: A novel quantitative method for functional characterization of human and murine platelet receptors. Clinical Chemistry and Laboratory Medicine . 2012;50(7):1253-1262. doi:10.1515/CCLM.2011.817

30. Greinacher A, Pecci A, Kunishima S, et al. Diagnosis of inherited platelet disorders on a blood smear: a tool to facilitate worldwide diagnosis of platelet disorders. Journal of Thrombosis and Haemostasis . 2017;15(7):1511-1521. doi:10.1111/jth.13729

31. Morozova DS, Martyanov AA, Korobkina JJD, et al. Granulocytes role in thrombus formation under low shear rate flow conditions as procoagulant platelet scavengers. Published online 2020.

32. Gracheva MA, Urnova ES, Sinauridze EI, et al. Thromboelastography, thrombin generation test and thrombodynamics reveal hypercoagulability in patients with multiple myeloma. Leukemia and Lymphoma . 2015;56(12):3418-3425. doi:10.3109/10428194.2015.1041385 
33. Mack JM, Verkamp B, Richter GT, Nicholas R, Stewart K, Crary SE. Effect of sirolimus on coagulopathy of slow-flow vascular malformations.Pediatr Blood Cancer . 2019;66(10):e27896. doi:10.1002/pbc.27896

34. Martyanov AA, Balabin FA, Dunster JL, Panteleev MA, Gibbins JM, Sveshnikova AN. Control of Platelet CLEC-2-Mediated Activation by Receptor Clustering and Tyrosine Kinase Signaling. Biophysical Journal . Published online April 2020:S0006349520303465. doi:10.1016/j.bpj.2020.04.023

35. Sveshnikova A. Hubs and Webs in Platelet Intracellular Signalling.SBPR . 2021;1(3):31-32. doi:10.52455/sbpr.01.202103014

36. Sveshnikova A, Stepanyan M, Panteleev M, Sveshnikova A, Stepanyan M, Panteleev M. Platelet functional responses and signalling: the molecular relationship. Part 1: responses. Systems Biology and Physiology Reports . 2021;1(1):20. doi:10.52455/sbpr.01.202101014

37. Folpe AL, Veikkola T, Valtola R, Weiss SW. Vascular endothelial growth factor receptor-3 (VEGFR-

3): A marker of vascular tumors with presumed lymphatic differentiation, including Kaposi's sarcoma, kaposiform and Dabska-type hemangioendotheliomas, and a subset of angiosarcomas. Modern Pathology . 2000;13(2):180-185. doi:10.1038/modpathol.3880033

\section{Legends}

Figure 1. Platelet responses to strong activation in KHE . (A) Platelet size and shape change (FSC-H mean fluorescence intensity (MFI)). (B) GPIIb/IIIa activation upon platelet stimulation measured by PAC1 binding. (C) Platelet GPIb shedding upon activation (resting GPIb ratio to activated GPIb). $(\mathrm{D}, \mathrm{E})$ Platelet $\alpha(\mathrm{D})$ and dense $(\mathrm{E})$ granule release upon activation. $(\mathrm{H})$ Percentage of annexin V-positive (procoagulant) platelets. Triangles correspond to healthy pediatric donors, circles correspond to patients with KHE/KMP. "CRP+TRAP-6" denotes activation with combination of $10 \mu \mathrm{g} / \mathrm{mL}$ CRP and $12.5 \mu \mathrm{M}$ SFLLRN. "2TR+ADP" denotes activation with combination of $12.5 \mu \mathrm{M}$ SFLLRN, $200 \mu \mathrm{M}$ AYGPKF and 2 $\mu \mathrm{M}$ ADP. Statistical significance was calculated using Mann-Whitney U-test, ${ }^{*}$ corresponds to $\mathrm{p}<0.05$.

Figure 2. Collagen-induced platelet thrombus formation under low shear rate. Thrombus area was measured after 5, 10 and 15 minutes of whole blood perfusion through fibrillar collagen-coated flow chamber. Thrombi were identified by fluorescence (highlighted in red). A - Thrombi of the thrombocytopenic patient with KMP (Patient 1). B - Thrombi of the typical non-thrombocytopenic patient with KHE. C Thrombi of the typical healthy pediatric donor. C - Comparison between thrombus areas in healthy controls and patients with KHE/KMP at different timepoints. Statistical significance was calculated using MannWhitney U-test, ${ }^{*}$ corresponds to $\mathrm{p}<0.05$.

Figure 3. Platelet intracellular signaling in KHE. A - Cytosolic calcium concentration in resting platelets, calcium increase upon stimulation with fucoidan $(100 \mu \mathrm{g} / \mathrm{mL})$ or rhodocytin $(200 \mathrm{nM})$, preincubation with $2 \mathrm{CP}$ and HB125 with or without sequential stimulation with $200 \mathrm{nM}$ rhodocytin. B,C Cytosolic calcium concentration (B) and fibrinogen binding (C) upon platelet stimulation with low doses of ADP $(2 \mu \mathrm{M})$, CRP $(2 \mu \mathrm{g} / \mathrm{mL})$ and TRAP-6 $(5 \mu \mathrm{M})$. Statistical significance was calculated using MannWhitney U-test, ${ }^{*}$ corresponds to $\mathrm{p}<0.05$.

Figure 4. Aggregation of the platelets of the patients with KHE is impaired. A - Typical aggregation curves for the LaSca assays upon activation with $800 \mathrm{nM}$ ADP (black) or $20 \mu \mathrm{g} / \mathrm{mL}$ collagen (red). B,C - Initial velocities of aggregation upon stimulation with ADP (B) were mostly diminished in patients with KHE, while maximal aggregation (C) was normal. D,E - Initial velocities of aggregation (D) and maximal aggregation upon stimulation with collagen were both decreased in most of the patients. F Initial velocity of platelet aggregation upon stimulation with $10 \mathrm{nM}$ rhodocytin was significantly decreased. $\mathrm{G}$ - Maximum platelet aggregation upon stimulation with $10 \mathrm{nM}$ rhodocytin was not affected by either of the inhibitors. ${ }^{*}$ represents $\mathrm{p}<0.05$ (the Mann-Whitney $\mathrm{U}$ test) 

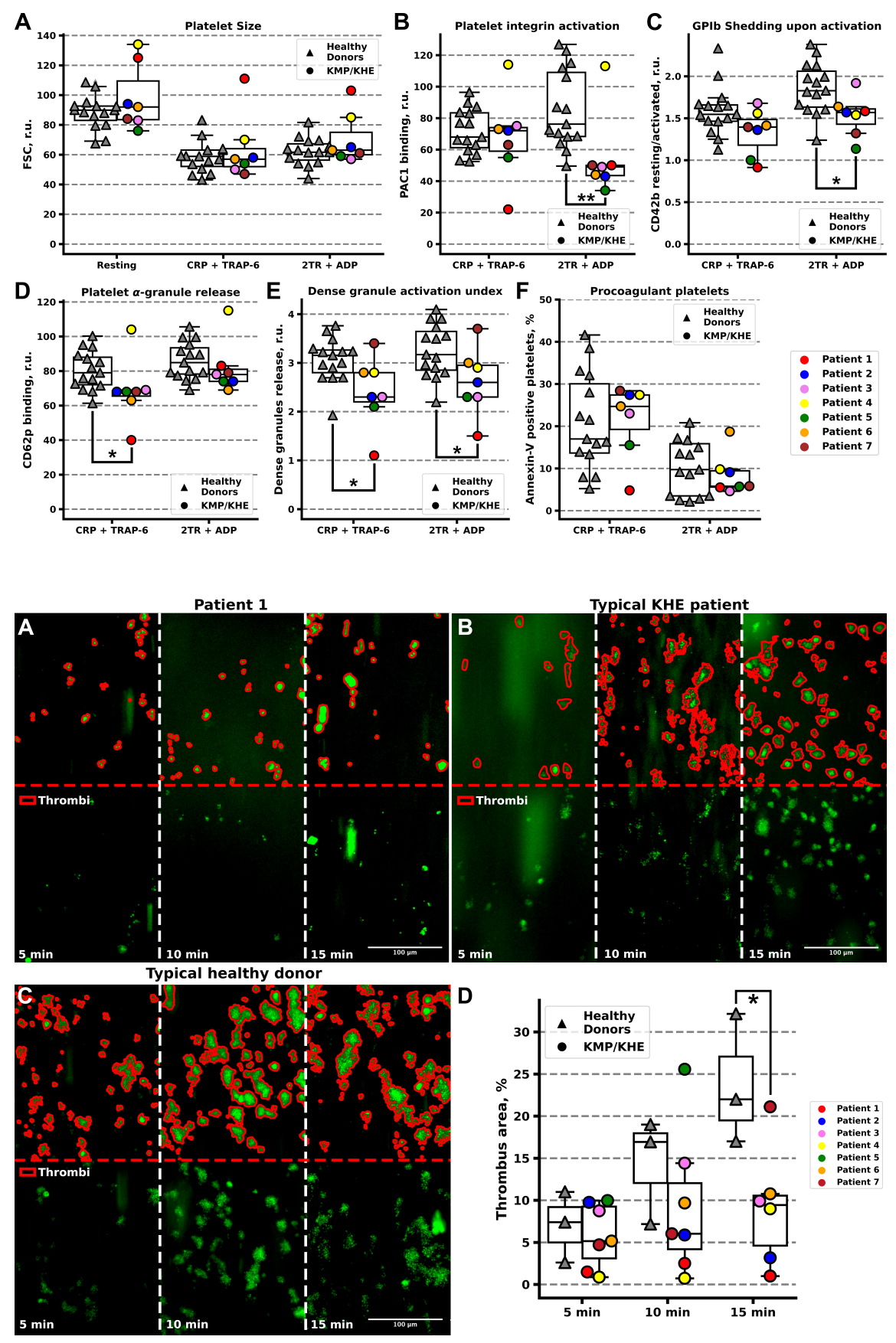

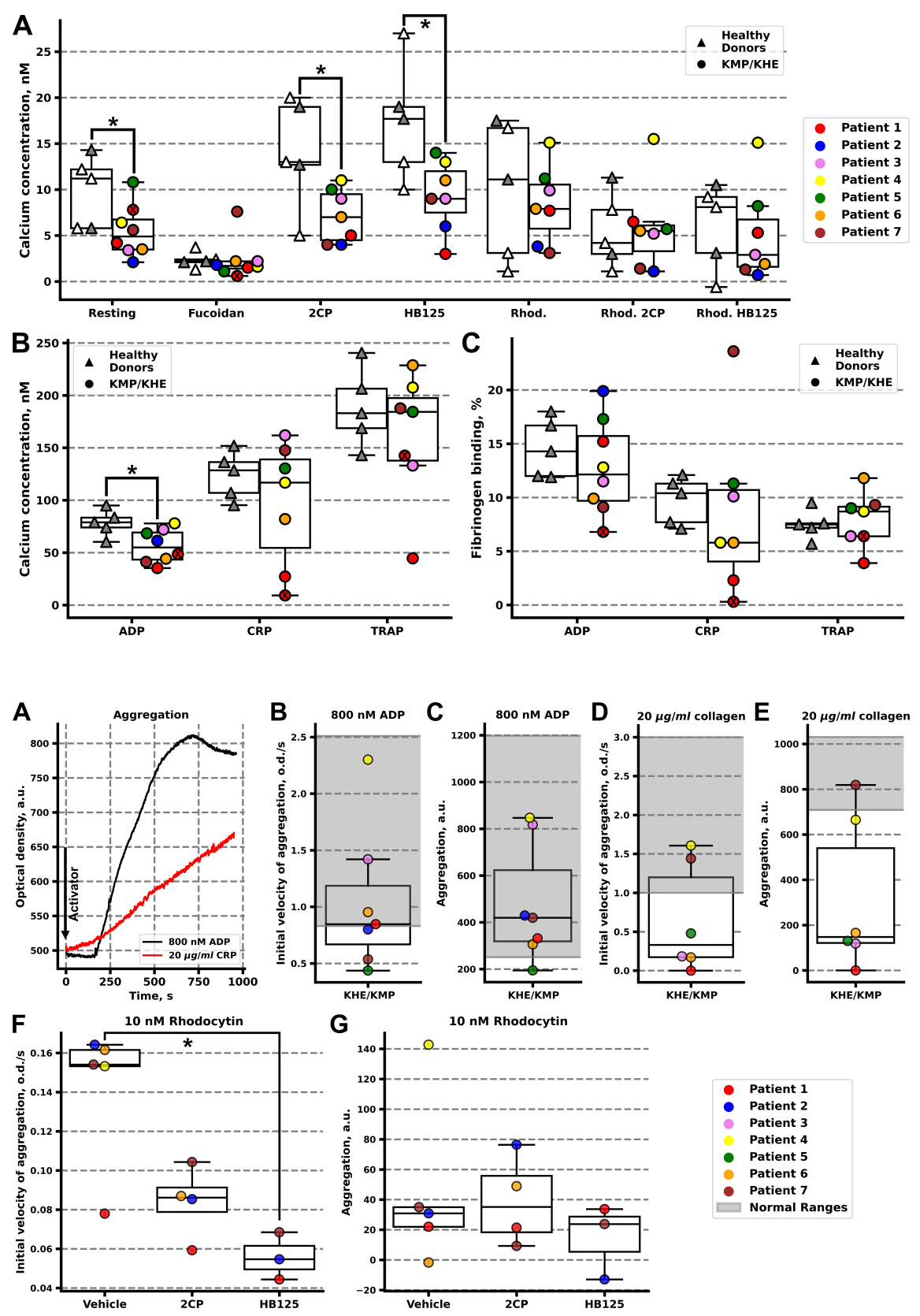